\title{
Errata: Stabilization of Superconductivity by Phase-Slip Centers in Current-Carrying Tin Whiskers*
}

\author{
R. Tidecks \\ I. Physikalisches Institut der Universität Göttingen, Göttingen, West Germany
}

(Received February 22, 1985)

The abstract should read:

The superconducting-normal transition in quasi-one-dimensional superconductors, such as whiskers, near the critical temperature is characterized by a large region of nonequilibrium superconductivity. Localized phase-slip centers lead to a voltage-step structure in the V-T and V-I characteristics. The width of the transition can be understood even by assuming the whisker to consist of very homogeneous material, because phase-slip centers stimulate the superconductivity in the whole sample, leading to an enhancement of the critical currents of other phase-slip centers. The present work reports quantitative measurements of this phenomenon and a discussion of possible explanations.

Footnote $c$ of Table I should read:

${ }^{c} \mathrm{ST}=d j_{c}^{2 / 3} / d T_{c}\left(\mu \mathrm{A} / \mu \mathrm{m}^{2}\right)^{2 / 3} \mathrm{~K}^{-1}$; the slope of the $j_{c}^{2 / 3}\left(T_{c}\right)$ straight line, where $j_{c}=I_{c} / A$ is the critical current density. 